\title{
DETERMINAÇÃO DO COMPORTAMENTO DE FASES A ALTA PRESSÃO DE SISTEMAS MULTICOMPONENTES CONTENDO EXTRATO DE BAGAÇO DE UVA SYRAH PARA COMPREENSÃO DO PROCESSO DE ENCAPSULAÇÃO A ALTA PRESSÃO
}

\author{
T.A.PROENÇA ${ }^{1}$, S.R.ROSSO ${ }^{1}$, D.A. OLIVEIRA ${ }^{1}$ e S.R.S.FERREIRA ${ }^{1}$ \\ ${ }^{1}$ Universidade Federal de Santa Catarina, Departamento de Engenharia Química e Engenharia de \\ Alimentos \\ E-mail para contato: sandra@enq.ufsc.br
}

\begin{abstract}
RESUMO - O bagaço de uva pode ser aproveitado para a obtenção de componentes antioxidantes, sensíveis à luz e ao ar. Por isso, o estudo de métodos de estabilização, tal como a co-precipitação com polímeros pela técnica SAS (Supercritical AntiSolvent) é necessário. O objetivo desse trabalho foi determinar o comportamento de fases do sistema acetato de etila +extrato de bagaço de uva $+\mathrm{CO}_{2}$, a fim de se obter a indicação de condições operacionais a serem empregadas em futuros ensaios SAS. Dados de equilíbrio de fases para o sistema foram coletados para composições variando de 50,0 \% a 96,0 \% em massa de $\mathrm{CO}_{2}$ nas temperaturas de $308 \mathrm{~K}, 313 \mathrm{~K}$ e $318 \mathrm{~K}$. Foram observadas transições de fase do tipo equilíbrio líquido-vapor. As condições sugeridas para realizar os ensaios de precipitação por SAS são: até $318 \mathrm{~K}$ para a preservação da qualidade do extrato, entre $90,0 \%$ e $95,0 \%$ em massa de $\mathrm{CO}_{2}$ para garantir a função de antissolvente do $\mathrm{CO}_{2}$ e acima de 95 bar para assegurar uma única fase para o sistema.
\end{abstract}

\section{INTRODUÇÃO}

Os compostos fenólicos estão amplamente distribuídos no reino vegetal e são uma importante categoria de fitoquímicos, devido à alta capacidade antioxidante e habilidade de eliminar radicais livres. Entre as frutas, a uva (Vitis vinifera) é uma das maiores fontes destes compostos, cujos principais representantes são os flavonóides (antocianinas e flavonóis), os estilbenos (como resveratrol), os ácidos fenólicos (derivados dos ácidos cinâmicos e benzóicos) e uma larga variedade de taninos (STUART et al., 2009).

O bagaço de uva, composto principalmente por engaço, semente e casca, representa um importante subproduto da indústria vinícola, uma vez que os compostos fenólicos permanecem no bagaço após a vinificação, em diferentes concentrações, dependendo do processo de fabricação e da variedade de uva (OLIVEIRA, 2010). Nesse sentido, a recuperação desses componentes de importância para as indústrias farmacêutica, química e de alimentos, pode ser uma alternativa para a valorização deste resíduo (SALES et al., 2012). 


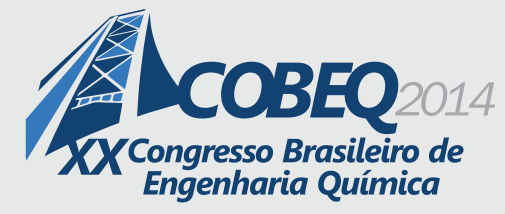

Os componentes antioxidantes presentes no bagaço de uva são sensíveis à luz e ao oxigênio. Por isso, torna-se necessário o estudo de métodos de estabilização, tal como a co-precipitação com polímeros pela técnica SAS (Supercritical AntiSolvent).

O uso da técnica SAS para processos de micronização e de encapsulamento tem sido proposto, visto que tal técnica promove a precipitação e encapsulamento de materiais sólidos com características controladas (tamanho e distribuição de tamanho, morfologia e estrutura cristalina) (MARTÍN et al., 2007). Estas características fornecidas pelo método SAS são devido à rápida mistura entre solvente, antissolvente e extrato, o que conduz a uma maior saturação do solvente e induz a formação de partículas de menores dimensões. Além disso, a separação eficiente do solvente e do antissolvente, evita resíduos de solvente no produto final e permite a reutilização do solvente e antissolvente, preservando a qualidade dos materiais particulados (BENELLI et al., 2014).

O conhecimento sobre o comportamento de fases de sistemas complexos formados por extratos de produtos naturais e solventes é muito importante para a compreensão do mecanismo de precipitação envolvido na formação de partículas (micronização) como a nucleação, a cinética do crescimento das partículas, e a transferência de massa. O entendimento do equilíbrio de fases também ajuda na determinação das condições de funcionamento satisfatório para os processos de precipitação e encapsulamento (FRANCESCHI et al., 2008).

Neste contexto, o objetivo desse estudo foi investigar o comportamento de equilíbrio de fases através do método estático-sintético para o sistema multicomponente composto por extrato de bagaço de Uva Syrah (Vitis vinifera), acetato de etila e dióxido de carbono supercrítico $\left(\mathrm{CO}_{2}\right)$. Estes dados termodinâmicos são importantes no estabelecimento de condições operacionais adequadas para futuros ensaios de coprecipitação com polímeros envolvendo a técnica SAS.

\section{MATERIAIS E MÉTODOS}

\subsection{Preparação das amostras e obtenção de extrato de bagaço de uva}

O resíduo do processamento de uvas Syrah (Vitis vinifera), composto por cascas, sementes e engaços, foi fornecido pela vinícola Ouro Verde (Vale do São Francisco/BA, Brasil) da empresa Miolo Wine Group.

A matéria-prima foi seca a $32{ }^{\circ} \mathrm{C}$ em estufa com circulação de ar (De Leo, Porto Alegre/RS, Brasil) até 10,1 \pm 0,3\% (w/w) do teor de umidade, alcançado após $20 \mathrm{~h}$ de secagem. O material seco foi moído em moinho de facas (De Leo, Porto Alegre / RS, Brasil) e caracterizado pela classificação do tamanho em uma peneira vibratória (Bertel metalúrgica Ind. Ltda., Caieiras / SP, Brasil). A fração de partículas de $-20 /+32$ malha foi selecionada para prosseguir as extrações.

O extrato de bagaço de uva foi obtido por extração Soxhlet, conforme descrito por Oliveira (2010). Resumidamente, coloca-se $5 \mathrm{~g}$ de amostra pré-tratada sobre o filtro colocado na câmara de extração suspensa acima do balão contendo o solvente e abaixo de um condensador. $\mathrm{O}$ balão contém $150 \mathrm{~mL}$ de acetato de etila e é aquecido por uma manta de aquecimento (Fisatom, Mod. 52 - Classe 


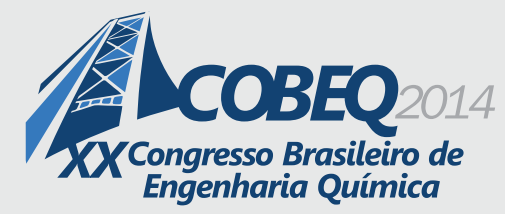

19 a 22 de outubro de 2014

Florianópolis/SC

300, São Paulo/SP, Brasil) na temperatura de ebulição do solvente, o qual evapora e se move na fase gasosa em direção ao condensador. Neste equipamento, o vapor é convertido em líquido, o qual goteja no filtro contendo a amostra. A câmara de extração é projetada de modo que quando o solvente em torno da amostra for superior à altura máxima do sifão, o líquido transborda para o balão onde é aquecido, e novamente evapora, completando um ciclo. Os ensaios foram realizados por $8 \mathrm{~h}$ e as misturas resultantes de soluto/solvente foram separadas por meio de um evaporador rotativo (Fisatom, modelo 802, São Paulo, Brasil), obtendo assim um extrato livre de solvente.

\subsection{Solução de extrato de bagaço de uva}

Para o equilíbrio de fases contendo acetato de etila, extrato de bagaço de uva e dióxido de carbono supercrítico foi preparado uma solução cuja proporção é $0,2452 \mathrm{~g}$ de extrato de bagaço de uva para $75 \mathrm{~mL}$ de acetato de etila.

\subsection{Aparelho de equilíbrio de fases e procedimento experimental}

Experimentos de equilíbrio de fases foram realizados através do método estático-sintético em uma célula de volume variável de alta pressão. O equipamento experimental e o procedimento adotados foram baseados nos estudos de Lanza (2004), Corazza (2002), Dariva (2000) e Stuart (1999). Em resumo, o equipamento é constituído por uma célula de equilíbrio, com o volume máximo interno de $27 \mathrm{ml}$ e duas janelas de safira (uma para entrada de luz e outra para a observação visual), um transdutor de pressão absoluta (Modelo 511 , Controlo Huba, Würenlos / Dinamarca) e uma bomba de seringa (260Cv Teledyne Isco, Lincoln / NE / EUA). As transições de fase, como consequência da manipulação de pressão (bomba de seringa), foram visualmente observadas (janelas de safira). Inicialmente, uma quantidade precisa da solução de extrato de bagaço de uva foi pesada numa balança analítica (Ohaus, AS200S modelo, NJ, EUA) com $\pm 0,0001 \mathrm{~g}$ de precisão e carregada na célula de equilíbrio. Uma quantidade conhecida de solvente a $5{ }^{\circ} \mathrm{C}$ e 100 bar foi carregada na célula de equilíbrio usando a bomba de seringa até se obter a fração de mássica desejada (de 50,0 \% a $\left.96,0 \% \mathrm{CO}_{2}\right)$. O conteúdo da célula foi mantido sob agitação contínua através de um agitador magnético. A temperatura da célula foi mantida constante com o uso de um banho termostático e na temperatura desejada, a pressão foi aumentada até à formação de um sistema com uma única fase. Neste ponto, a pressão foi diminuída lentamente (a uma taxa de 3,5 bar / min) até a formação incipiente de uma nova fase. Este processo foi repetido pelo menos duas vezes para cada temperatura (308 K, 313 K e 318 K) e composição global testada.

\section{RESULTADOS E DISCUSSÃO}

Dados de equilíbrio de fases obtidos para o sistema multicomponente extrato do bagaço de uva+ acetato de etila $+\mathrm{CO}_{2}$ são apresentados na Tabela 1 para o intervalo de temperatura de $308-318 \mathrm{~K}$ e a composição em massa de $\mathrm{CO}_{2}(\mathrm{w})$ de 50,0 \% a 96,0 \%. A Tabela 1 mostra os resultados em termos de equilíbrio de pressão de transição e apresenta o erro experimental associado para cada condição representada pelo desvio padrão das medições repetidas $(\sigma)$. O diagrama de pressão-composição (Pw) para os resultados apresentados na Tabela 1 é apresentado na Figura 1. 
Tabela 1- Dados experimentais de equilíbrio de fases para o sistema extrato do bagaço de uva+ acetato de etila $+\mathrm{CO}_{2}$

\begin{tabular}{|c|c|c|c|c|c|c|c|}
\hline $\mathbf{T}(\mathrm{K})$ & P (Bar) & $\sigma($ Bar $)$ & Transição & $\mathbf{T}(\mathrm{K})$ & P (Bar) & $\sigma$ (Bar) & Transição \\
\hline \multicolumn{4}{|c|}{$w C O_{2}: 0,4979$} & \multicolumn{4}{|c|}{$w \mathrm{CO}_{2}: 0,9302$} \\
\hline 308 & & & & 308 & 68,8 & 0,4 & ELV-PB \\
\hline 313 & 50,3 & 0,5 & ELV-PB & 313 & 77,6 & 0,1 & ELV-PB \\
\hline 318 & 65,7 & 0,3 & ELV-PB & 318 & 82,7 & 0,1 & ELV-PB \\
\hline \multicolumn{4}{|c|}{$w C O_{2}: 0,8002$} & \multicolumn{4}{|c|}{$w C O_{2}: 0,9453$} \\
\hline 308 & 68,8 & 0,1 & ELV-PB & 308 & 75,5 & 0,2 & ELV-PB \\
\hline 313 & 71,6 & 0,3 & ELV-PB & 313 & 79,8 & 0,3 & ELV- PB \\
\hline 318 & 76,9 & 0,1 & ELV-PB & 318 & 83,6 & 0,1 & ELV-PB \\
\hline \multicolumn{4}{|c|}{${ }_{w C O}: 0,8498$} & \multicolumn{4}{|c|}{$w \mathrm{CO}_{2}: 0,9599$} \\
\hline 308 & 71,2 & 0,2 & ELV-PB & 308 & 81,7 & 0,3 & ELV-PB \\
\hline 313 & 74,3 & 0,5 & ELV-PB & 313 & 86,5 & 0,2 & ELV-PB \\
\hline 318 & 79,6 & 0,3 & ELV-PB & 318 & 91,1 & 0,1 & ELV- PB \\
\hline \multicolumn{4}{|c|}{$w C O_{2}: 0,8962$} & & & & \\
\hline 308 & 69,0 & 0,4 & ELV-PB & & & & \\
\hline 313 & 76,0 & 0,1 & ELV-PB & & & & \\
\hline 318 & 79,0 & 0,4 & ELV-PB & & & & \\
\hline
\end{tabular}

Como pode ser verificado na Tabela 1, e a partir do diagrama de pressão/composição (Figura 1), as medidas experimentais revelaram um comportamento de fases simples para o sistema multicomponente formado por extrato de bagaço de uva + acetato de etila $+\mathrm{CO}_{2}$, que consistiu apenas na ocorrência do equilíbrio do tipo líquido-vapor (ELV).

Para as isotermas entre $308 \mathrm{~K}$ e $318 \mathrm{~K}$, Figura 1, a região acima das curvas denota o sistema em uma única fase, enquanto que abaixo da isoterma observa-se a região de equilíbrio líquido-vapor.

Observa-se que tanto o aumento da temperatura quanto o da concentração de $\mathrm{CO}_{2}$ afetam bastante as pressões de transição. Isso se deve ao aumento da energia interna com o aumento da temperatura. Por extensão, é necessária uma pressão maior para que o sistema apresente-se totalmente miscível, principalmente quando as isotermas se aproximam do ponto crítico da mistura, onde os efeitos energéticos entre os componentes são relevantes (BENDER, 2008). 


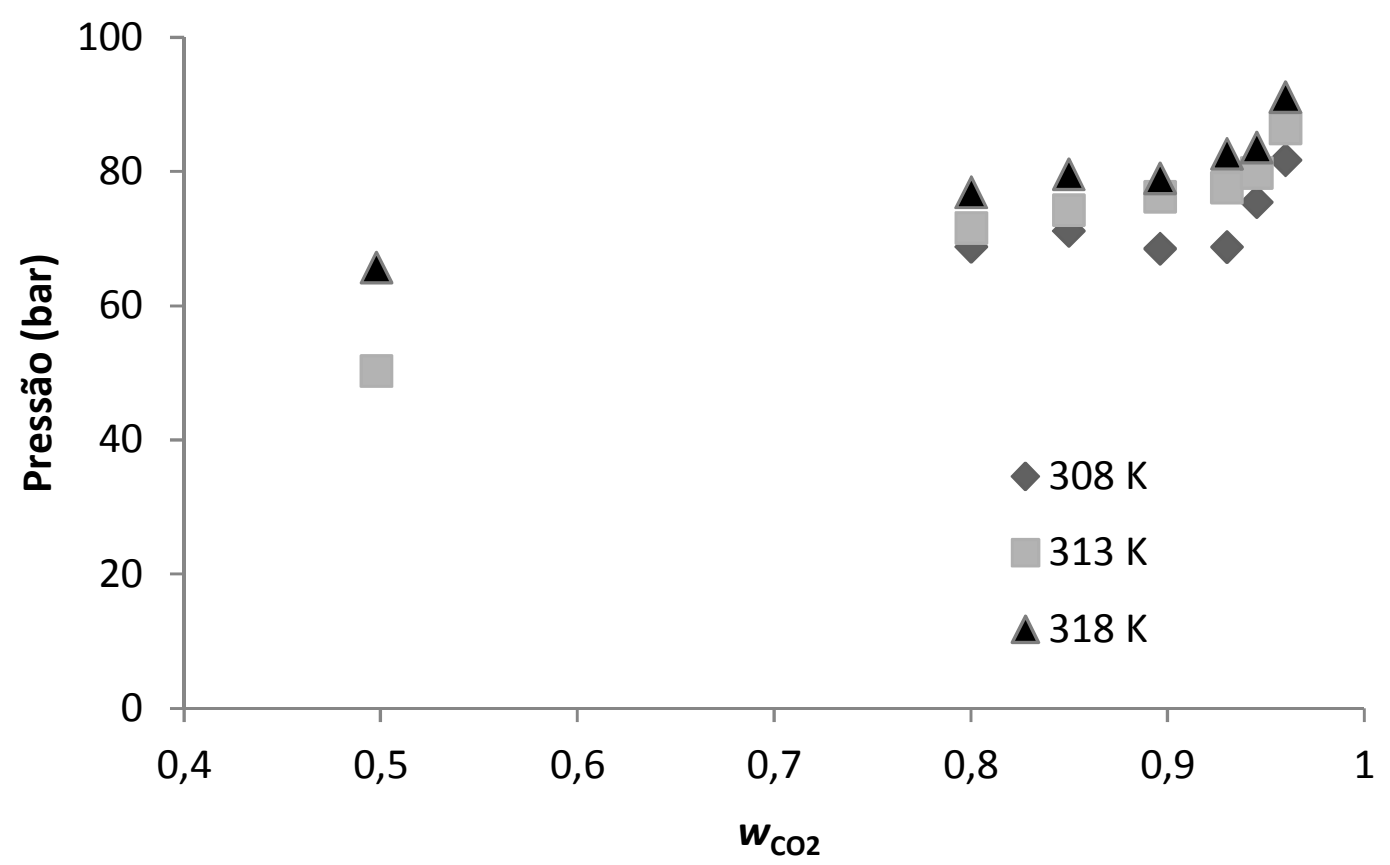

Figura 1- Diagrama (P-w) do sistema multicomponente $\mathrm{CO}_{2}+$ extrato de bagaço de uva a $308 \mathrm{~K}$, $313 \mathrm{~K}$ e $318 \mathrm{~K}$.

A Figura 1 permite selecionar adequadamente as condições de precipitação de partículas por meio de técnica de SAS, usando dióxido de carbono como anti-solvente e acetato de etila como solvente. O ponto de operação sugerido para realização dos experimentos do método SAS encontra-se acima do ponto crítico da mistura. Esta condição operacional assegura a miscibilidade completa entre o solvente e o anti-solvente e é a condição recomendada para a obtenção de micropartículas (BENELLI et al., 2014). A condição de única fase tende a evitar o congelamento do extrato que resultaria na formação filamentos devido à rápida separação das fases na coluna de precipitação.

Neste contexto, para garantir que ensaios de precipitação e/ou encapsulamento por SAS sejam realizados em uma região homogênea, em que os componentes entrem no sistema completamente miscíveis, e numa região de alta concentração de $\mathrm{CO}_{2}$ para garantir que ele atue como anti-solvente, as condições de temperatura, pressão e composição sugeridas são: até $318 \mathrm{~K}$ para a preservação da qualidade do extrato, entre $90,0 \%$ e $95,0 \%$ em massa de $\mathrm{CO}_{2}$ para garantir a função de antissolvente do $\mathrm{CO}_{2}$ e acima de 95 bar para assegurar uma única fase para o sistema.

\section{CONCLUSÕES}

$\mathrm{O}$ comportamento de fase do sistema de extrato de bagaço de uva + acetato de etila $+\mathrm{CO}_{2}$ foi investigado para temperaturas de 308-318 K e frações mássicas de $\mathrm{CO}_{2}$ variando de 50 a $96 \%$. Os dados de equilíbrio de fases foram registrados para pressões de até 91,1 bar, permitindo a detecção de 


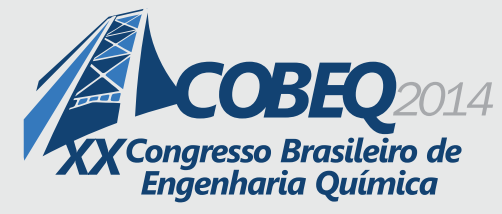

transição do tipo líquido-vapor. Esses dados obtidos são úteis para definir as condições de temperatura, pressão e composição mássica de $\mathrm{CO}_{2}$ para os processos de precipitação ou encapsulamento do extrato de bagaço de uva, aplicando o processo SAS.

\section{REFERÊNCIAS BIBLIOGRÁFICAS}

BENDER, J. P. Equilíbrio de fases de polímeros biocompatíveis e monômeros: dados experimentais e modelagem. Dissertação de mestrado, departamento de ciências agrárias, Programa de mestrado em Engenharia de alimentos, URI-Campus de Erechim, 2008.

BENELLI, P., COMIM, S. R. R.; OLIVEIRA, J. V.; PEDROSA, R. C.; FERREIRA, S. R. S. Phase equilibrium data of guac, atonga (Casearia sylvestris)extract + ethanol + CO2system and encapsulation using a supercriticalanti-solvent process. The Journal of Supercritical Fluids, p. 1-9. 2014.

CORAZZA, M. L., Equilíbrio de Fases do Sistema Reacional para a Oxidação do Limoneno em CO2SC. Dissertação de Mestrado (Mestre em Ciências em Engenharia Química) - Programa de Mestrado em Engenharia Química - Universidade Estadual de Maringá (DEQ/UEM), Maringá - PR, 2002.

DARIVA, C., Equilíbrio de Fases a Altas Pressões em Sistemas com Prolipropilenos. Dados Experimentais e Modelagem SAFT. Tese de Doutorado (Doutor em Ciências em Engenharia Química) - Programa de Engenharia Química PEQ - Universidade Federal do Rio de Janeiro (COPPE/UFRJ), Rio de Janeiro - RJ, 2000.

FRANCESCHI, E.; CESARO, A. C; FEITEN, M.; FERREIRA, S.R.S.; DARIVA, C.; KUNITA, M.. H.; RUBIRA, A. F.; MUNIZ, E. C.; CORAZZA, M. L.; OLIVEIRA, J. V. Precipitation of $\beta$-carotene and PHBV and co-precipitation from SEDS technique using supercritical $\mathrm{CO}_{2}$. J. of Supercritical Fluids. p. 259-269. 2008.

LANZA, M. Comportamento de fases dos óleos de oliva, soja e mamona em n-butano e propano a alta pressão. Dissertacao de mestrado, Departamento de Ciencias Agrarias, Programa de Mestrado em Engenharia de Alimentos, URI- Campus de Erechim, 2004.

MARTÍN, A.; MATTEA, F.; GUTIÉRREZ, L.; MIGUEL, F.; COCERO, M. J. Co-precipitation of carotenoids and bio-polymers with the supercritical anti-solvent process. J. of Supercritical Fluids, p. 138-147. 2007.

OLIVEIRA, D. A. Caracterização Fitoquímica e Biológica de Extratos Obtidos de Bagaço de Uva (Vitis Vinifera) das Variedades Merlot e Syrah. 2010. 209 f. Dissertação (Mestrado) - Curso de Engenharia de Alimentos, Universidade Federal de Santa Catarina, Florianópolis, 2010.

SALES, N. F. F.; CRUZ1, A. P. G.; CABRAL, L. M. C.; TORRES, A. G. Capacidade Antioxidante de Extratos Hidroalcoólicos do Bagaço de Uva Tinta. In: Congresso Brasileiro de Engenharia Química. Búzios: 2012. p. 11435 - 11441. Disponível em: <http://ainfo.cnptia.embrapa.br/digital/bitstream/item/70742/1/2012-189.pdf>. Acesso em: 27 mar. 


\section{ACoseQ XXCongresso Brasileiro de Engenharia Química}

19 a 22 de outubro de 2014

Florianópolis/SC

2014.

STUART, G. R., Estudo do Fracionamento do Óleo de Laranja por Destilação à Vácuo e Dióxido de Carbono Supercrítico. Tese de Doutorado, (Doutor em Ciências em Engenharia Química) - Programa de Engenharia Química PEQ - Universidade Federal do Rio de Janeiro (COPPE/UFRJ), Rio de Janeiro - RJ, 1999. 